\title{
A note on the existence of the power investor's optimizer
}

\author{
Kasper Larsen \\ Department of Mathematical Sciences, \\ Carnegie Mellon University, \\ Pittsburgh, PA 15213 \\ kasperl@andrew. cmu.edu
}

February 2, 2009

\begin{abstract}
[KLSX91] ensure the existence of the expected utility maximizer for investors with constant relative risk aversion coefficients less than one. In this note, we explain a simple trick that allows us to use this result to provide the existence of utility maximizers for arbitrary coefficients of relative risk aversion. The simplicity of our approach is to be contrasted with the general existence result provided in [KS99].
\end{abstract}

Key Words: Incomplete markets, convex duality, change of measure, expected utility. 


\section{Introduction}

The problem of optimal investment in continuous time has a long history in mathematical finance dating back to Merton's seminal works. The papers [CH89] and [KLS87] introduce martingale techniques and provide the existence of the expected utility maximizer in the complete Brownian setting. [KLSX91] use Hilbert space analysis to provide the existence of the utility optimizer in the general incomplete Brownian setting for certain utility functions whereas [KS99] use more sophisticated tools from functional analysis to provide the general existence result.

The investor's preferences are often modeled by a power function, $U(x) \triangleq$ $\frac{x^{p}}{p}$, where $1-p$ is referred to as the constant relative risk aversion parameter. The set of utility specifications covered in [KLSX91] includes $p \in(0,1)$ but not $p<0$. Our main contribution is a basic change-of-measure argument, which provides an existence proof for $p<0$ via the results in [KLSX91]. The simplicity of our approach is to be compared with the general duality analysis developed in [KS99].

[KLSX91] phrase the dual problem as an unconstrained Hilbert space minimization problem. We link the dual problem to a fictitious market and provide a Hilbert space in which the fictitious dual unconstrained optimizer can be located in the case $p<0$. We thereby provide the foundation to construct a numerical algorithm based on unconstrained numerical Hilbert space techniques which can numerically compute the investor's optimizer.

All stochastic processes are defined on a finite time horizon $[0, T], T>0$, and on a common filtered probability space $(\Omega, \mathcal{F}, \mathbb{F}, \mathbb{P})$ where $\mathbb{F} \triangleq\left\{\mathcal{F}_{t}\right\}_{t \in[0, T]}$ is the standard completed filtration generated by a two dimensional Brownian motion $(B, W)$ and we take $\mathcal{F} \triangleq \mathcal{F}_{T} . \quad \mathcal{L}^{k}, k=1,2$, denotes the set of progressively measurable processes $\theta$ for which $\mathbb{P}\left(\int_{0}^{T}\left|\theta_{u}\right|^{k} d u<\infty\right)=1$ and we note that $\mathcal{L}^{k}$ is invariant under equivalent measure changes. $\mathbb{L}^{2}(\mathbb{P} \times$ Leb), respectively $\mathbb{L}^{2}(\mathbb{P})$, denotes the set of square integrable progressively measurable processes, respectively $\mathcal{F}_{T}$-measurable square integrable random variables. For $\theta \in \mathcal{L}^{2}$ and a Brownian motion $M$, we define the Doléans-Dade exponential as

$$
\mathcal{E}_{t}^{M}(\theta) \triangleq \exp \left(\int_{0}^{t} \theta_{u} d M_{u}-\frac{1}{2} \int_{0}^{t} \theta_{u}^{2} d u\right), \quad t \in[0, T] .
$$




\section{The underlying model}

We assume that the financial market consists of only two securities: a numéraire security $S^{(0)}, S_{t}^{(0)} \triangleq 1, t \in[0, T]$, and a risky security $S$ with dynamics

$$
d S_{t} \triangleq S_{t}\left(\mu_{t} d t+\sigma_{t} d B_{t}\right), \quad t \in(0, T], \quad S_{0} \triangleq 1
$$

Since $W$ can appear in the dynamics of $\mu$ and $\sigma$, this model specification is incomplete. Everything that follows can be generalized to a financial market consisting of $n$ securities driven by a $d$-dimensional Brownian motion.

We will focus on the set of non-negative wealth processes and the following assumption ensures that there are no arbitrage opportunities in this set. Furthermore, it places us in the exact same setting as used in [KLSX91].

Assumption 2.1. Both the volatility process $\sigma, \sigma>0$, and the market price of risk process $\lambda_{t} \triangleq \frac{\mu_{t}}{\sigma_{t}}$ are in $\mathcal{L}^{2}$. In this case the drift process $\mu$ is in $\mathcal{L}^{1}$.

A process $\pi$ is deemed an admissible portfolio fraction process if $\pi$ satisfies the properties $\pi \mu \in \mathcal{L}^{1}$ and $\pi \sigma \in \mathcal{L}^{2}$ in which case we write $\pi \in \mathcal{A}$. We note that under Assumption 2.1, Cauchy-Schwartz's inequality ensures that $\pi \sigma \in \mathcal{L}^{2}$ implies $\pi \mu \in \mathcal{L}^{1}$; hence, under Assumption 2.1, the set $\mathcal{A}$ only depends on $\sigma$. The investor's wealth dynamics $\left(X_{t}^{x, \pi}\right)_{t}$ are specified in terms of admissible portfolio fractions $\pi \in \mathcal{A}$ invested in $S$ :

$$
d X_{t}^{x, \pi} \triangleq X_{t}^{x, \pi} \pi_{t}\left(\mu_{t} d t+\sigma_{t} d B_{t}\right), \quad t \in(0, T], \quad X_{0}^{x, \pi} \triangleq x
$$

where $x>0$ denotes the investor's initial wealth. The investor's preferences are modeled by a power function, $U(x) \triangleq \frac{1}{p} x^{p}$ for $p \in(-\infty, 0) \cup(0,1)$. The investor is assumed to maximize expected utility of terminal wealth over admissible fraction processes:

$$
u(x) \triangleq \sup _{\pi \in \mathcal{A}} \mathbb{E}\left[U\left(X_{T}^{x, \pi}\right)\right], \quad x>0 .
$$

Given the power utility assumption, we note that any optimal fraction $\hat{\pi} \in \mathcal{A}$ for the primal problem (2.1) is independent of the investor's initial wealth $x$.

\section{Primal-dual relations}

For $p \in(-\infty, 0) \cup(0,1)$, the dual problem associated with the primal problem (2.1) is defined by

$(3.1) v(y) \triangleq \inf _{\nu \in \mathcal{L}^{2}} \mathbb{E}\left[V\left(y \mathcal{E}_{T}^{B}(-\lambda) \mathcal{E}_{T}^{W}(\nu)\right)\right], V(y) \triangleq \frac{y^{-q}}{q}, q \triangleq \frac{p}{1-p}, y>0$. 
As in the primal problem (2.1), we note that any dual optimizer $\hat{\nu} \in \mathcal{L}^{2}$ is independent of the initial value $y>0$. The following result is basically Theorem 9.4(i) in [KLSX91], which is stated under the assumption that $U(0)>-\infty$. As noted in the textbook [KS98], Theorem 6.4.1, this assumption is not needed for the validity of the result. This result is also embedded in the general analysis of [KS99]. However, given that our investor is modeled by power utility, we can present a short and self contained proof.

Lemma 3.1. Under Assumption 2.1, $p \in(-\infty, 0) \cup(0,1)$ and $v(1)<\infty$ : If there exists $\hat{\nu} \in \mathcal{L}^{2}$ attaining the infimum in (3.1) (in particular, $v$ is finitely valued), then $u(x)<\infty$ for all $x>0$ and there exists a fraction process $\hat{\pi} \in \mathcal{A}$ attaining the supremum in the primal problem (2.1).

Proof: We let $\hat{\nu} \in \mathcal{L}^{2}$ be the dual minimizer attaining the infimum in (3.1). For $\pi \in \mathcal{A}$, Fenchel's inequality gives us the weak duality relation

$$
U\left(X_{T}^{x, \pi}\right) \leq V\left(\mathcal{E}_{T}^{B}(-\lambda) \mathcal{E}_{T}^{W}(\hat{\nu})\right)+X_{T}^{x, \pi} \mathcal{E}_{T}^{B}(-\lambda) \mathcal{E}_{T}^{W}(\hat{\nu}), \quad \mathbb{P} \text {-a.s. },
$$

and the supermartingale property of the last term then shows $u(x)<\infty$.

For $\nu \in \mathcal{L}^{2}$ and $\epsilon \in(0,0.5)$, we define the process $\nu^{\epsilon} \in \mathcal{L}^{2}$ by

$$
\nu_{t}^{\epsilon} \triangleq \frac{(1-\epsilon) \hat{\nu}_{t} \mathcal{E}_{t}^{W}(\hat{\nu})+\epsilon \nu_{t} \mathcal{E}_{t}^{W}(\nu)}{(1-\epsilon) \mathcal{E}_{t}^{W}(\hat{\nu})+\epsilon \mathcal{E}_{t}^{W}(\nu)}, \quad t \in[0, T] .
$$

For $t \in[0, T], \nu^{\epsilon}$ satisfies $\mathcal{E}_{t}^{W}\left(\nu^{\epsilon}\right)=(1-\epsilon) \mathcal{E}_{t}^{W}(\hat{\nu})+\epsilon \mathcal{E}_{t}^{W}(\nu) \rightarrow \mathcal{E}_{t}^{W}(\hat{\nu}), \mathbb{P}$-a.s. as $\epsilon \downarrow 0$. We then have by $\hat{\nu}$ 's optimality and secondly by $V$ 's convexity:

$$
\begin{aligned}
0 & \leq \frac{1}{\epsilon} \mathbb{E}\left[V\left(\mathcal{E}_{T}^{B}(-\lambda) \mathcal{E}_{T}^{W}\left(\nu^{\epsilon}\right)\right)-V\left(\mathcal{E}_{T}^{B}(-\lambda) \mathcal{E}_{T}^{W}(\hat{\nu})\right)\right] \\
& \leq \mathbb{E}\left[\left(\mathcal{E}_{T}^{B}(-\lambda) \mathcal{E}^{W}\left(\nu^{\epsilon}\right)\right)^{-q-1}\left(\mathcal{E}_{T}^{B}(-\lambda) \mathcal{E}_{T}^{W}(\hat{\nu})-\mathcal{E}_{T}^{B}(-\lambda) \mathcal{E}_{T}^{W}(\nu)\right)\right] .
\end{aligned}
$$

Furthermore, for all $\epsilon \in(0,0.5)$ we have the estimate

$$
\left(\mathcal{E}_{T}^{B}(-\lambda)\right)^{-q}\left(\mathcal{E}_{T}^{W}\left(\nu^{\epsilon}\right)\right)^{-q-1} \mathcal{E}_{T}^{W}(\hat{\nu}) \leq 0.5^{-q-1}\left(\mathcal{E}_{T}^{B}(-\lambda) \mathcal{E}_{T}^{W}(\hat{\nu})\right)^{-q}, \quad \mathbb{P} \text {-.a.s. }
$$

which is integrable since $v(1)<\infty$. We can therefore use the dominated convergence theorem on the first term in (3.2). Since the second term in (3.2) is negative, we can use Fatou's lemma when passing $\epsilon$ to zero. All in all, by applying $\lim \sup _{\epsilon \downarrow 0}$ on both sides of the estimate (3.2) we obtain

$$
0 \leq \mathbb{E}\left[\left(\mathcal{E}_{T}^{B}(-\lambda) \mathcal{E}_{T}^{W}(\hat{\nu})\right)^{-q-1}\left(\mathcal{E}_{T}^{B}(-\lambda) \mathcal{E}_{T}^{W}(\hat{\nu})-\mathcal{E}_{T}^{B}(-\lambda) \mathcal{E}_{T}^{W}(\nu)\right)\right] .
$$


It follows that $\hat{X} \triangleq\left(\mathcal{E}_{T}^{B}(-\lambda) \mathcal{E}_{T}^{W}(\hat{\nu})\right)^{-q-1}$ satisfies the conditions of Theorem 8.5 in [KLSX91] for the initial wealth $x \triangleq \mathbb{E}\left[\hat{X} \mathcal{E}_{T}^{B}(-\lambda) \mathcal{E}_{T}^{W}(\hat{\nu})\right]=q v(1) \in$ $(0, \infty)$ and thereby a replicating fraction process $\hat{\pi} \in \mathcal{A}$ for $\hat{X}$ is provided. Theorem 9.3 in [KLSX91] then shows that $\hat{X}$ is the optimal terminal wealth and given the power utility assumption, $\hat{\pi}$ is independent of the initial wealth $x$, which concludes the proof.

In the next section we will also need the following observation:

Lemma 3.2. Under Assumption 2.1: For $p \in(0,1)$ and $u(x)<\infty$ for all $x>0$ (equivalently for some $x>0$ ), we have $v(y)<\infty$ for all $y>0$.

Proof: We define the bounded stopping times $\left(\tau^{(n)}\right)_{n}$ as follows

$$
\tau^{(n)} \triangleq \inf \left\{t: \int_{0}^{t} \lambda_{u}^{2} d u=n\right\} \wedge T, \quad n \in \mathbb{N} .
$$

We then define the assets $\left(S^{(n)}\right)_{n}$ by

$$
d S_{t}^{(n)} \triangleq S_{t}^{(n)}\left(\mu_{t} 1_{\left\{t \leq \tau^{(n)}\right\}} d t+\sigma_{t} d B_{t}\right), \quad t \in(0, T], \quad S_{0}^{(n)} \triangleq 1, \quad n \in \mathbb{N} .
$$

The market price of risk process $\lambda^{(n)}$ corresponding to $S^{(n)}$ is given by $\lambda_{t}^{(n)} \triangleq \lambda_{t} 1_{\left\{t \leq \tau^{(n)}\right\}}$. We let $u^{(n)}$ and $v^{(n)}$ denote the corresponding value functions. Since $S^{(n)}$ 's volatility term is $\sigma$, we note that the set of admissible fractions corresponding to $S^{(n)}$ is by Assumption 2.1 also the set $\mathcal{A}$. By $\tau^{(n)}$ 's construction, we have $\mathbb{E}\left[V\left(\mathcal{E}_{T}^{B}\left(-\lambda^{(n)}\right)\right)\right]<\infty$; hence, $v^{(n)}(1)<\infty$.

First we prove $u^{(n)}(x) \leq u(x)$ for all $n \in \mathbb{N}$ and $x>0$. To see this, we let $\pi^{(n)} \in \mathcal{A}$ be arbitrary and denote by $X^{x, \pi^{(n)}}$ the corresponding wealth process. Since $\pi^{(n)} \in \mathcal{A}$, we can define the strictly positive supermartingale $Y_{t} \triangleq \mathcal{E}_{t}^{B}\left(\pi^{(n)} \sigma\right), t \in[0, T]$. The optional sampling theorem shows that $\mathbb{E}\left[Y_{T} \mid \mathcal{F}_{\tau^{(n)}}\right] \leq Y_{\tau^{(n)}}$. Finally, we define $\pi_{t} \triangleq \pi_{t}^{(n)} 1_{\left\{t \leq \tau^{(n)}\right\}} \in \mathcal{A}$ and we denote by $X^{x, \pi}$ the corresponding wealth process. We then have the following relation $\mathbb{P}$-.a.s.

$$
X_{T}^{x, \pi^{(n)}}=X_{T}^{x, \pi} \exp \left(\int_{\tau^{(n)}}^{T} \pi_{u}^{(n)} \sigma_{u} d B_{u}-\frac{1}{2} \int_{\tau^{(n)}}^{T}\left(\pi_{u}^{(n)} \sigma_{u}\right)^{2} d u\right)=X_{T}^{x, \pi} \frac{Y_{T}}{Y_{\tau^{(n)}}} .
$$

Since $X_{T}^{x, \pi}=X_{\tau^{(n)}}^{x, \pi}$, iterated expectations and Jensen's inequality give us

$$
\begin{aligned}
\mathbb{E}\left[U\left(X_{T}^{x, \pi^{(n)}}\right)\right] & =\mathbb{E}\left[U\left(X_{T}^{x, \pi}\right) \mathbb{E}\left[\left(\frac{Y_{T}}{Y_{\tau^{(n)}}}\right)^{p} \mid \mathcal{F}_{\tau^{(n)}}\right]\right] \\
& \leq \mathbb{E}\left[U\left(X_{T}^{x, \pi}\right) \mathbb{E}\left[\left(\frac{Y_{T}}{Y_{\tau^{(n)}}}\right) \mid \mathcal{F}_{\tau^{(n)}}\right]^{p}\right] \leq \mathbb{E}\left[U\left(X_{T}^{x, \pi}\right)\right] \leq u(x),
\end{aligned}
$$


showing that $u^{(n)}(x) \leq u(x)$ for all $n \in \mathbb{N}$.

Since $v^{(n)}(1)<\infty$, we can use Theorem 12.3 in [KLSX91] to produce $\hat{\nu}^{(n)} \in \mathbb{L}^{2}(\mathbb{P} \times$ Leb) such that

$$
v^{(n)}(1)=\inf _{\nu \in \mathcal{L}^{2}} \mathbb{E}\left[V\left(\mathcal{E}_{T}^{B}\left(-\lambda^{(n)}\right) \mathcal{E}_{T}^{W}(\nu)\right)\right]=\mathbb{E}\left[V\left(\mathcal{E}_{T}^{B}\left(-\lambda^{(n)}\right) \mathcal{E}_{T}^{W}\left(\hat{\nu}^{(n)}\right)\right)\right] .
$$

We will now show that the sequence $\left(\hat{\nu}^{(n)}\right)_{n}$ is norm bounded in $\mathbb{L}^{2}(\mathbb{P} \times$ Leb), i.e., $\sup _{n}\left\|\hat{\nu}^{(n)}\right\|_{2}<\infty$. From Proposition 11.4, equation (11.9) in [KLSX91], we have

$$
v^{(n)}(1)=\sup _{x>0}\left\{u^{(n)}(x)-x\right\} \leq \sup _{x>0}\{u(x)-x\}<\infty,
$$

by assumption. Since $p \in(0,1)$ the mapping $y \rightarrow V\left(e^{y}\right)$ is convex; hence,

$$
v^{(n)}(1) \geq V\left(\exp \left(-\frac{1}{2}\left(\left\|\lambda^{(n)}\right\|_{2}^{2}+\left\|\hat{\nu}^{(n)}\right\|_{2}^{2}\right)\right)\right)
$$

by Jensen's inequality. Furthermore, since $y \rightarrow V\left(e^{y}\right)$ is non-increasing, (3.3) and (3.4) show that the sequences

$$
V\left(\exp \left(-\frac{1}{2}\left(\left\|\lambda^{(n)}\right\|_{2}^{2}\right)\right)\right), \quad V\left(\exp \left(-\frac{1}{2}\left(\left\|\hat{\nu}^{(n)}\right\|_{2}^{2}\right)\right)\right), \quad n \in \mathbb{N}
$$

are uniformly bounded. The property $V(0)=\infty$ then provides the norm boundedness of both sequences $\left(\hat{\nu}^{(n)}\right)_{n}$ and $\left(\lambda^{(n)}\right)_{n}$. Therefore, we see by the monotone convergence theorem

$$
\infty>\lim _{n \rightarrow \infty}\left\|\lambda^{(n)}\right\|_{2}^{2}=\lim _{n \rightarrow \infty} \mathbb{E}\left[\int_{0}^{\tau^{(n)}} \lambda_{u}^{2} d u\right]=\mathbb{E}\left[\int_{0}^{T} \lambda_{u}^{2} d u\right],
$$

since $\tau^{(n)} \uparrow T, \mathbb{P}$-.a.s., for $n \rightarrow \infty$. A similar argument shows that $\| \lambda^{(n)}-$ $\lambda \|_{2} \rightarrow 0$ for $n \rightarrow \infty$. The norm boundedness of $\left(\hat{\nu}^{(n)}\right)_{n}$ and the reflexivity of $\mathbb{L}^{2}\left(\mathbb{P} \times\right.$ Leb) allow us to extract a weakly convergent subsequence of $\left(\hat{\nu}^{(n)}\right)_{n}$. Mazur's lemma then allows us to find a double array of non-negative numbers $\alpha_{k}^{(n)}, k=n, \ldots, K(n), K(n) \in \mathbb{N}$, such that

$$
\sum_{k=n}^{K(n)} \alpha_{k}^{(n)}=1, \quad n \in \mathbb{N}, \quad \text { and } \quad \tilde{\nu}^{(n)} \triangleq \sum_{k=n}^{K(n)} \alpha_{k}^{(n)} \hat{\nu}^{(k)} \rightarrow \tilde{\nu} \text { in } \mathbb{L}^{2}(\mathbb{P} \times \text { Leb }) .
$$


We also have $\tilde{\lambda}^{(n)} \triangleq \sum_{k=n}^{K(n)} \alpha_{k}^{(n)} \lambda^{(k)} \rightarrow \lambda$ in $\mathbb{L}^{2}(\mathbb{P} \times$ Leb) and therefore $\mathcal{E}_{T}^{B}\left(-\tilde{\lambda}^{(n)}\right) \mathcal{E}_{T}^{W}\left(\tilde{\nu}^{(n)}\right) \rightarrow \mathcal{E}_{T}^{B}(-\lambda) \mathcal{E}_{T}^{W}(\tilde{\nu})$ in probability. Since $q>0, y \rightarrow V\left(e^{y}\right)$ is convex and non-increasing, which together with Fatou's lemma yield

$$
\begin{aligned}
v(1) & \leq \mathbb{E}\left[V\left(\mathcal{E}_{T}^{B}(-\lambda) \mathcal{E}_{T}^{W}(\tilde{\nu})\right)\right] \leq \liminf _{n} \mathbb{E}\left[V\left(\mathcal{E}_{T}^{B}\left(-\tilde{\lambda}^{(n)}\right) \mathcal{E}_{T}^{W}\left(\tilde{\nu}^{(n)}\right)\right)\right] \\
& \leq \liminf _{n} \sum_{k=n}^{K(n)} \alpha_{k}^{(n)} \mathbb{E}\left[V\left(\mathcal{E}_{T}^{B}\left(-\lambda^{(k)}\right) \mathcal{E}_{T}^{W}\left(\hat{\nu}^{(k)}\right)\right)\right]=\liminf _{n} \sum_{k=n}^{K(n)} \alpha_{k}^{(n)} v^{(k)}(1),
\end{aligned}
$$

which is finite by (3.3).

\section{Main existence result for $p<0$.}

This section contains our main contribution. For $p<0$, we have $q \triangleq \frac{p}{1-p} \in$ $(-1,0)$; hence, under Assumption 2.1 we have $\mathbb{E}\left[\left(\mathcal{E}_{T}^{B}(-\lambda)\right)^{-q}\right] \in(0,1)$. We can then introduce the strictly positive martingale $\xi$ and the probability measure $\tilde{\mathbb{P}}$ :

$$
\xi_{t} \triangleq \frac{\mathbb{E}\left[\left(\mathcal{E}_{T}^{B}(-\lambda)\right)^{-q} \mid \mathcal{F}_{t}\right]}{\mathbb{E}\left[\left(\mathcal{E}_{T}^{B}(-\lambda)\right)^{-q}\right]}, \quad t \in[0, T], \quad \frac{d \tilde{\mathbb{P}}}{d \mathbb{P}} \triangleq \xi_{T} .
$$

The martingale representation theorem provides a pair of processes $\phi^{B}, \phi^{W} \in$ $\mathcal{L}^{2}$ such that

$$
d \xi_{t}=\xi_{t}\left(\phi_{t}^{B} d B_{t}+\phi_{t}^{W} d W_{t}\right), \quad t \in[0, T] .
$$

Furthermore, Girsanov's theorem ensures

$$
\tilde{B}_{t} \triangleq B_{t}-\int_{0}^{t} \phi_{u}^{B} d u, \quad \tilde{W}_{t} \triangleq W_{t}-\int_{0}^{t} \phi_{u}^{W} d u, \quad t \in[0, T],
$$

are independent Brownian motions under the measure $\tilde{\mathbb{P}}$. Finally, we introduce the artificial dual value function for $p<0$ defined by

$$
\tilde{v}(y) \triangleq \inf _{\nu \in \mathcal{L}^{2}} \tilde{\mathbb{E}}\left[\tilde{V}\left(y \mathcal{E}_{T}^{\tilde{W}}\left(-\phi^{W}\right) \mathcal{E}_{T}^{\tilde{B}}(\nu)\right)\right], \quad \tilde{V}(y) \triangleq \frac{y^{p}}{-p}, \quad y>0,
$$

where $\tilde{\mathbb{E}}[C]$ denotes the expectation of a random variable $C$ under the probability measure $\tilde{\mathbb{P}}$. Our existence proof hinges on the following observation: 
Lemma 4.1. Under Assumption 2.1: For $p<0$ and $\tilde{v}(y)<\infty$, we can find $\hat{\nu} \in \mathbb{L}^{2}(\tilde{\mathbb{P}} \times$ Leb) attaining the infimum in (4.1).

PROOF: The following is a slight modification of the proof of Lemma 12.8 in [KLSX91]. Since $\tilde{v}(1)<\infty$ we can find $\nu^{(0)} \in \mathcal{L}^{2}$ such that

$$
\tilde{\mathbb{E}}\left[\tilde{V}\left(\mathcal{E}_{T}^{\tilde{W}}\left(-\phi^{W}\right) \mathcal{E}_{T}^{\tilde{B}}\left(\nu^{(0)}\right)\right)\right]<\infty .
$$

We then define the bounded stopping times $\left(\tau^{(n)}\right)_{n}$ as follows

$$
\tau^{(n)} \triangleq \inf \left\{t: \int_{0}^{t}\left(\left(\phi_{u}^{W}\right)^{2}+\left(\nu_{u}^{(0)}\right)^{2}\right) d u=n\right\} \wedge T, \quad n \in \mathbb{N} .
$$

The mapping $\tilde{V}$ is convex and non-increasing; hence, by the optional sampling theorem and Jensen's inequality we have the inequality

$$
\tilde{\mathbb{E}}\left[\tilde{\mathbb{E}}\left[\tilde{V}\left(\mathcal{E}_{T}^{\tilde{W}}\left(-\phi^{W}\right) \mathcal{E}_{T}^{\tilde{B}}\left(\nu^{(0)}\right)\right)\right] \mathcal{F}_{\tau^{(n)}}\right] \geq \tilde{\mathbb{E}}\left[\tilde{V}\left(\mathcal{E}_{\tau^{(n)}}^{\tilde{W}}\left(-\phi^{W}\right) \mathcal{E}_{\tau^{(n)}}^{\tilde{B}}\left(\nu^{(0)}\right)\right)\right] .
$$

Furthermore, for $p<0$, we have $y \rightarrow \tilde{V}\left(e^{y}\right)$ is convex and so by another application of Jensen's inequality the above right-hand-side dominates

$$
\tilde{V}\left(\exp \left(-\frac{1}{2} \mathbb{E}\left[\int_{0}^{\tau^{(n)}}\left(\left(\phi_{u}^{W}\right)^{2}+\left(\nu_{u}^{(0)}\right)^{2}\right) d u\right]\right)\right) .
$$

We can then use the monotone convergence theorem when passing $n$ to infinity; hence, (4.2) and $\tilde{V}(0)=\infty$ show that $\phi^{W}$ (and $\nu^{(0)}$ ) must be in $\mathbb{L}^{2}(\tilde{\mathbb{P}} \times$ Leb $)$. The result now follows from Theorem 12.3 in [KLSX91]; the remaining assumptions of this theorem are satisfied because $p<0$.

Our goal is to link this existence in the artificial dual problem (4.1) to the existence in the original dual problem (3.1) when $p<0$ and then subsequently provide the maximizer for the primal problem (2.1) using Lemma 3.1.

Theorem 4.2. Under Assumption 2.1: For $p<0$, there exists a fraction process $\hat{\pi} \in \mathcal{A}$ attaining the supremum in the original primal problem (2.1).

Proof: We define the artificial security $\tilde{S}$ by

$$
d \tilde{S}_{t} \triangleq \tilde{S}_{t}\left(d \tilde{W}_{t}+\phi_{t}^{W} d t\right), \quad t \in(0, T], \quad \tilde{S}_{0} \triangleq 1 .
$$


$\tilde{\mathcal{A}}$ denotes the corresponding admissible fractions and since $\phi^{W} \in \mathcal{L}^{2}$, CauchySchwartz's inequality shows $\tilde{\mathcal{A}}=\mathcal{L}^{2}$. For $\nu \in \tilde{\mathcal{A}}$, the wealth dynamics are

$$
d \tilde{X}_{t}^{x, \nu}=\tilde{X}_{t}^{x, \nu} \nu_{t}\left(d \tilde{W}_{t}+\phi_{t}^{W} d t\right), \quad t \in(0, T], \quad \tilde{X}_{0}^{x, \nu}=x,
$$

and we note that $\mathcal{E}_{t}^{W}(\nu)=\tilde{X}_{t}^{1, \nu}$. The artificial primal problem corresponding to the artificial dual problem (4.1) is given by (here we use $\tilde{\mathcal{A}}=\mathcal{L}^{2}$ ):

$$
\tilde{u}(x) \triangleq \sup _{\nu \in \mathcal{L}^{2}} \tilde{\mathbb{E}}\left[\tilde{U}\left(\tilde{X}_{T}^{x, \nu}\right)\right], \tilde{U}(x) \triangleq \frac{x^{-q}}{-q}, x>0, q \triangleq \frac{p}{1-p} \in(-1,0) .
$$

We can now re-write the original dual problem (3.1) in terms of (4.3) and then subsequently in terms of (4.1). Since $q \triangleq \frac{p}{1-p}<0$ we have

$$
\begin{aligned}
v(1) & =\inf _{\nu \in \mathcal{L}^{2}} \frac{1}{q} \mathbb{E}\left[\left(\mathcal{E}_{T}^{B}(-\lambda) \mathcal{E}_{T}^{W}(\nu)\right)^{-q}\right] \\
& =\inf _{\nu \in \mathcal{L}^{2}} \frac{1}{q} \mathbb{E}\left[\left(\mathcal{E}_{T}^{B}(-\lambda)^{-q}\right] \tilde{\mathbb{E}}\left[\left(\mathcal{E}_{T}^{W}(\nu)\right)^{-q}\right]\right. \\
& =-\mathbb{E}\left[\left(\mathcal{E}_{T}^{B}(-\lambda)\right)^{-q}\right] \sup _{\nu \in \mathcal{L}^{2}} \frac{1}{-q} \tilde{\mathbb{E}}\left[\left(\mathcal{E}_{T}^{W}(\nu)\right)^{-q}\right] \\
& =-\mathbb{E}\left[\left(\mathcal{E}_{T}^{B}(-\lambda)\right)^{-q}\right] \tilde{u}(1) .
\end{aligned}
$$

Since $q \in(-1,0)$, the original dual value function $v$ given by (3.1) is finitely valued; hence, $\tilde{u}(1)<\infty$. Lemma 3.2 shows that $\tilde{v}(y)<\infty$ and the existence of a minimizer for the artificial dual problem (4.1) follows from Lemma 4.1. Lemma 3.1 (or Theorem 9.4(i) in [KLSX91]) then provides the optimizer $\hat{\nu} \in \tilde{\mathcal{A}}=\mathcal{L}^{2}$ for the artificial primal problem (4.3), which by construction is the minimizer for the original dual problem (3.1). Applying Lemma 3.1 again yields the optimizer for the original primal problem (2.1).

\section{References}

[CH89] Cox, J. C., and C. Huang (1989): Optimal Consumption and Portfolio Policies when Asset Prices follow a Diffusion Process, J. Econom. Theory 49, 33-83.

[KLS87] Karatzas, I., J. P. Lehoczky and S. E. Shreve (1987): Optimal Portfolio and Consumption Decisions for a "Small Investor" on a Finite Horizon, SIAM J. Control Optim. 25, 1557-1586. 
[KLSX91] Karatzas, I., J. P. Lehoczky, S. E. Shreve and G. L. Xu (1991): Martingale and Duality Methods for Utility Maximization in an Incomplete Market, SIAM J. Control Optim. 29, 707-730.

[KS98] Karatzas, I., and S. E. Shreve (1998): Methods of Mathematical Finance, Springer.

[KS99] Kramkov, D., and W. Schachermayer (1999): The Asymptotic Elasticity of Utility Functions and Optimal Investment in Incomplete Markets, Ann. Appl. Prob. 9, 904-950. 